\title{
Enhancement of perceptual representations by endogenous attention biases competition in response selection
}

\author{
Jane Klemen • Frederick Verbruggen • \\ Corrina Skelton • Christopher D. Chambers
}

Published online: 9 August 2011

(C) Psychonomic Society, Inc. 2011

\begin{abstract}
Perception and response selection are core processes in the generation of overt behavior. Selective attention is known to facilitate behavioral performance by altering perceptual processes. It remains unclear, however, whether selective attention can aid the resolution of response conflict, and if so, at what stage of processing this takes place. In two experiments, an endogenous cuing task was combined with a flanker task to assess the interaction of selective attention with response selection. The results of Experiment 1 show that cuing reduces the flanker-congruency effect when the cue and flanker are presented in close temporal proximity to each other. The results of Experiment 2 demonstrate that pre- but not post-cuing the target reduced the congruency effect, showing that selective attention can affect performance, but is ineffective once stimulus processing has proceeded to response selection. Our results provide evidence that selective attention can aid the resolution of response conflict by altering early perceptual processing stages.
\end{abstract}

Keywords Attention: Selective · Perception and Action

Electronic supplementary material The online version of this article (doi:10.3758/s13414-011-0188-5) contains supplementary material, which is available to authorized users.

J. Klemen $(\bowtie) \cdot$ C. Skelton $\cdot$ C. D. Chambers

School of Psychology and Cardiff University Brain Research

Imaging Centre (CUBRIC), Cardiff University,

Tower Building, Park Place,

Cardiff CF10 3AT, UK

e-mail: klemenj@cardiff.ac.uk

F. Verbruggen

Psychology, University of Exeter,

Washington Singer Laboratories, Streatham Campus,

Exeter EX4 4QG, UK
The ability to interact with objects in our environment is an important everyday skill. Without this ability, we would be unable to drive a car or pick up a coffee mug. It is commonly assumed that there are three main stages of information processing that underlie interactions with the environment: stimulus perception, response selection, and response execution (Sternberg, 1969). Each of these stages is necessary, since an object must be identified and a response toward the object chosen in order for a response to be carried out. Successful behavior requires mechanisms to selectively attend to task-relevant stimuli, and to resolve competition between different response options. The cognitive system is unable to process all of the information that is available in the environment at once, and mechanisms of selective attention ensure prioritization of goal-relevant information over irrelevant information (e.g., Deutsch \& Deutsch, 1963; Lavie, 1995; Treisman, 1969). Similarly, during response-selection stages, conflict might arise between goal-relevant responses and goal-irrelevant responses (e.g., Kornblum, Hasbroucq \& Osman, 1990; Ridderinkhof, 2002). This might also involve prioritization of the goal-relevant information. The central question addressed in the present studies was how selective visual attention assists in the resolution of response competition.

Competition during response selection is often studied with flanker paradigms (Eriksen \& Eriksen, 1974) in which participants respond to a target stimulus while ignoring any surrounding task-irrelevant stimuli. If the flankers are associated with a different response (incongruent flankers) than that appropriate for the target stimulus, task performance is poorer as compared with when the target is flanked by neutral distractors that are not associated with a response (e.g., Eriksen \& Eriksen, 1974; Eriksen \& Schultz, 1979; Ridderinkhof \& van der Molen, 1995). This flanker effect (FE) has been explained using the continuous flow 
model (Eriksen \& Schultz, 1979), according to which processing of the irrelevant flanker stimuli occurs in parallel with processing of the target. When flankers and targets indicate different responses, competition arises at the response-selection stage. This competition needs to be resolved before the target response can be executed (Eriksen \& Schultz, 1979; Smith \& Ratcliff, 2004). The flanker cost reflects the time needed to resolve the competition. In some situations, a flanker benefit has been observed when the flankers and the target lead to the same response: Studies have reported that RTs are shorter for congruent flankers than for neutral flankers (see Flowers, 1990, for a discussion of this). This benefit is possibly due to the target and the flanker activating the same response (Eriksen \& Schultz, 1979).

The distinction we draw between perceptual selection (i.e., attentional selection of stimuli) and response selection follows an established body of work that has demonstrated the separability of these processes via, for example, the psychological refractory period (Pashler, 1994) or via flanker paradigms that isolate the behavioral consequences of response conflict (Chambers et al., 2007; Hazeltine, Bunge, Scanlon \& Gabrieli 2003). Flanker tasks provide a measure of response selection by varying the congruence of responses triggered by targets versus distractors, independent of their perceptual salience or task relevance. Incongruent trials generate response conflict, and the unique performance cost associated with incongruent versus neutral trials gauges the additional processing required for resolving these competing action plans. Flanker tasks thus provide a measure of response selection that goes over and above the involuntary attentional capture by the distractor.

On the basis of modeling work, it has been proposed that the flanker cost can be reduced by selectively attending to the target location - that is, by altering perceptual processes (Cohen, Servan-Schreiber \& McClelland, 1992). Whether selective attention can indeed have an impact on response selection was the focus of a key study by Ro, Machado, Kanwisher and Rafal (2002). The authors combined a cuing paradigm with a modified version of the flanker task. Cuing paradigms are used to study attentional selection (Posner, 1980; Posner \& Cohen, 1984) and typically require participants to fixate centrally and to respond to peripheral targets. Prior to the target presentation, a central (endogenous) or peripheral (exogenous) cue is presented, which guides the participants' attention to the location at which the target will appear. As compared with uncued trials, participants typically respond faster to cued targets. This demonstrates that performance benefits from spatially directing selective attention prior to responding to target stimuli (e.g., Posner, 1980; Posner \& Cohen, 1984).

Ro et al. (2002) combined such a cuing paragraph with a flanker task that required the participants to respond to a red or green color patch. A flanker was concurrently presented and was mapped on to a congruent (same color) or incongruent (different color) response. In Experiments $1-3$, the authors cued the flanker location rather than the target location and assumed that directing attention to the flankers would increase the flanker cost. However, they found that neither endogenously nor exogenously directing attention to the flanker location at short or long cue-target stimulus onset asynchronies (SOAs) significantly influenced the flanker effect. The authors also report a nonsignificant effect for their Experiment 4, in which attention was exogenously directed to the target location. These findings led the authors to conclude that neither endogenous (informative) nor exogenous (uninformative) cuing of spatial attention interacts with response selection in the flanker task.

However, several other studies suggest that under some circumstances, selective attention can influence the flanker effect. Paquet and Lortie (1990) compared experimental blocks in which a fixation point at the target location of a flanker task was present with blocks in which it was absent, thus manipulating the certainty about the target location. It was found that presenting a fixation point and effectively cuing the target location reduced the flanker effect by half. Consistent with the idea of Cohen et al. (1992), this finding suggests that selective attention can influence response competition. Paquet and Lortie argued that under the condition of positional uncertainty (no fixation point), participants find it difficult to correctly focus their attention on the target location. Consequently, attention spreads to the flanker locations, thus enhancing flanker processing and, hence, the flanker effect. Under the condition of positional certainty (fixation point present), however, early selection processes reduce, if not eliminate, the processing of the flankers. This will result in a reduction of the flanker effect. Huckauf and Heller (2002, Experiment 2) examined whether cues following the target could also be effective in reducing the flanker effect. The authors found that precuing the target location by 50 and $100 \mathrm{~ms}$, as well as postcuing by $50 \mathrm{~ms}$, significantly reduced the flanker effect, as measured by error rates, in comparison with no cue and a cue presented simultaneously with the target.

Ro et al.'s (2002) null results for all interactions of cuing with response selection might have been due to several factors. First, the target location was always known in their study. Locational certainty about the target location has been shown to reduce the flanker effect (Paquet \& Lortie, 1990) and could therefore reduce the effect of cuing. Second, only one flanker was used. It is known that larger numbers of flankers produce larger flanker effects (Chastain, Cheal \& Lyon, 1996). Thus, the flanker effects reported by Ro and colleagues might have been too small to be changed significantly by cuing. Third, the cuing effect observed in Ro et al.'s experiments might have been submaximal and 
therefore less likely to interact with the flanker effect. Cues in Ro et al.'s study were never fully informative. Previous research has reported that the effect of a cue in a search task increases monotonically with the degree of information provided by the cue (Jonides, 1980; Klein, 2004). Thus, Ro et al. possibly did not induce a strong enough cuing effect to show a statistically significant interaction between cuing and the flanker effect. Fourth, Ro et al. tested only simultaneous target-flanker presentations. Some studies have also shown that timing might be critical in the flanker task. Many studies have shown that the flanker effect is largest when $\mathrm{SOA}=<0 \mathrm{~ms}$ (i.e., the flankers precede the target), and that it decreases when SOA $>100$ ms (Eriksen \& Schultz, 1979; Flowers, 1980; Flowers, 1990; Flowers \& Wilcox, 1982; Grice, Boroughs \& Canham, 1984; Taylor, 1977; Wascher, Reinhard, Wauschkuhn \& Verleger 1999). Thus, although the literature suggests that Ro and colleagues should have observed a cuing effect with simultaneous target-flanker presentation, it is possible that attention interacts with the flanker effect only at certain target-flanker SOAs. Fifth, possibly the limited number of observations per participant (mostly 24 trials per condition) resulted in insufficient experimental power. Finally, for Experiments 1-3, the flanker rather than the target location was cued, which has been shown to reduce the flanker effect (Munneke, Van der Stigchel \& Theeuwes, 2008).

In the present studies, we examined the temporal dynamics of how endogenous selective attention has an impact on response selection. We combined an informative endogenous cuing task with a flanker task in two experiments. In Experiment 1, we asked whether attentional cuing modulates response competition when flankers and targets are presented at a range of different SOAs. In Experiment 2, we further probed the time course of the interaction between selective attention and response competition by presenting cues after the target. In particular, we asked whether attention aids the resolution of response conflict by focusing perceptual processes or by modulating response selection mechanisms.

\section{Experiment 1}

In Experiment 1, we adopted a similar flanker task with varying target-flanker SOA, as was used by Eriksen and Schultz (1979), but included an endogenous cue 1,000 ms prior to the target in order to study how selective attention would influence response competition. We anticipated that we would replicate Eriksen and Schultz' findings concerning the target-flanker SOA. Additionally, we predicted that cuing the target location would improve performance by decreasing spatial uncertainty about the target location (Paquet \& Lortie, 1990). Whether such a cuing benefit would be observed at all target-flanker SOAs was uncertain.
Method

Participants Eighteen participants, of the ages 20-33 years ( $M=23.8, S D=3.6$, nine females, nine males), were paid for their participation. All participants were right-handed, had normal or corrected-to-normal vision, and were naive to the purpose of the experiment. Participants gave their informed written consent. The experiment was approved by the School of Psychology Ethics Committee of Cardiff University.

Stimuli, apparatus, and procedure The experiment was conducted in a dark room. Visual stimuli were presented on a 21-in. CRT monitor $(1,024 \times 768$ pixel resolution) at a refresh rate of $60 \mathrm{~Hz}$, with participants seated at a $65 \mathrm{~cm}$ viewing distance. Throughout the experiment, the participants' heads were fixed using a chin- and forehead rest, and eye movements were recorded using a $250-\mathrm{Hz}$ video eyetracker (Cambridge Research Systems, UK).

The stimuli consisted of a modified version of the Eriksen and Schultz (1979) flanker task combined with a spatial cuing paradigm (Posner \& Cohen, 1984). The trial sequence is depicted in Fig. 1. A black background with four circular, peripheral gray placeholders remained present throughout the experiment, indicating the four possible target locations (Fig. 2). The placeholders surrounded a central fixation point. Target stimuli were monochrome sine wave gratings (100 ms duration, 3.3 cycles/degree, $100 \%$ contrast, Fig. 2b). Participants responded to the orientation of a target stimulus (horizontal or vertical) by pressing the "1" or " 2 " buttons of the numerical keypad with their right hand index and middle fingers, respectively. The stimulusresponse mapping was counterbalanced across participants. The target stimuli always appeared within one of the four gray placeholders and consisted of either a vertical or a horizontal circular grating. A further four stimuli were shown outside of the four gray placeholders, thus "flanking" the target stimulus (Fig. 2a). These flankers could either be congruent (same grating orientation as the target), incongruent (opposite grating orientation of the target), or neutral (a plaid made up of the horizontal and vertical stimuli, which had no associated response, Fig. 2b).

Each experimental trial began with a central fixation point. After $200 \mathrm{~ms}$, a cue appeared at fixation for $500 \mathrm{~ms}$. This cue was either an arrowhead, which indicated the location the target was going to appear in, or a "star," which acted as a neutral cue. The arrowhead cue was always fully informative, thus indicating the correct location of the target $100 \%$ of the time. The neutral cue indicated only that the flankers and target were about to be presented, thus ensuring the same temporal warning in both the cued and uncued con- 


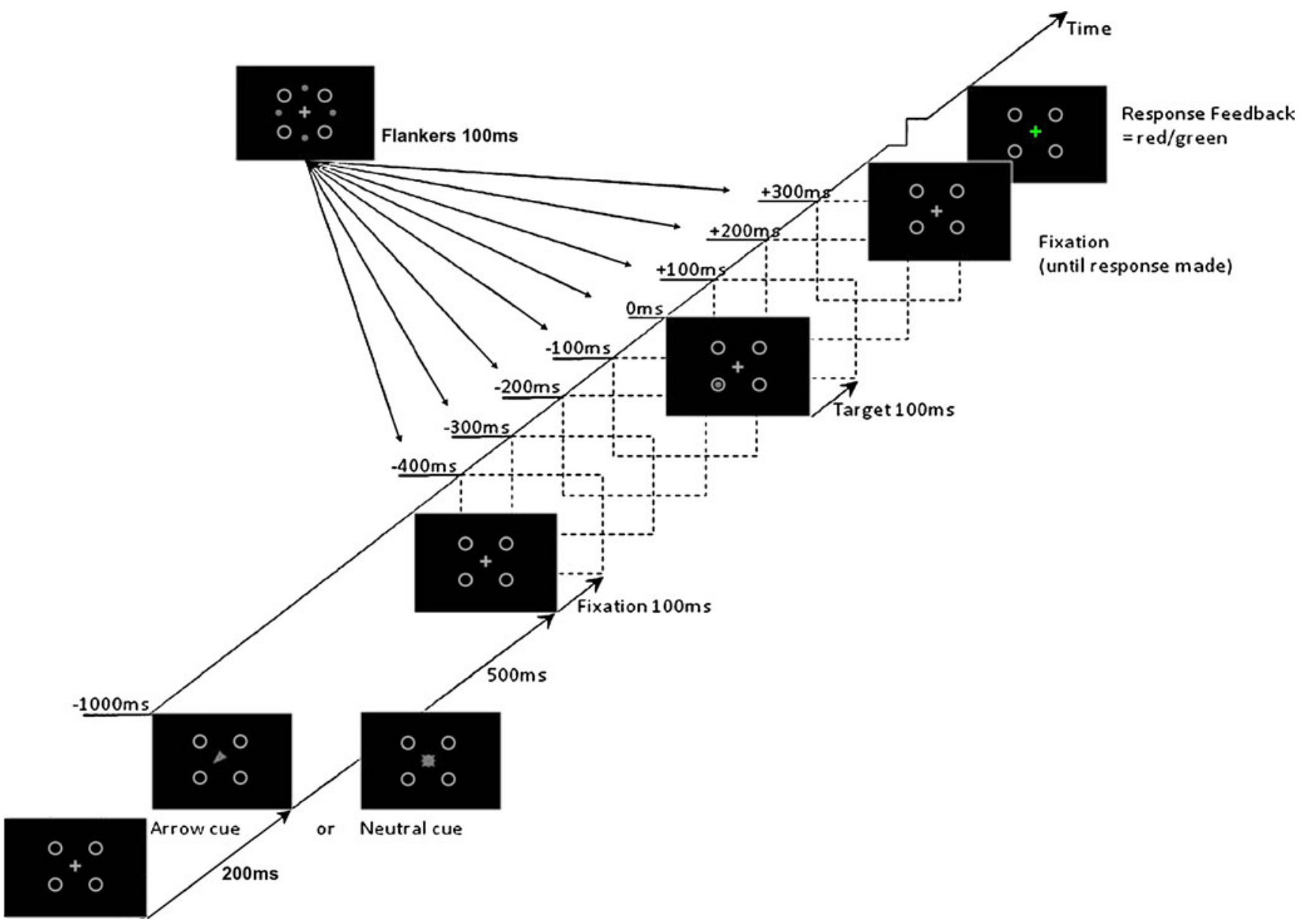

Fig. 1 Stimulus sequence for the flanker task employed in Experiment 1. See main text for details

ditions. The interval between the cue onset and target onset was always the same $(1,000 \mathrm{~ms})$

The cue was replaced by a fixation cross after $500 \mathrm{~ms}$. The fixation cross remained on the screen throughout the trial until a response was registered. The target appeared on the screen $500 \mathrm{~ms}$ after the presentation of the fixation cross. The flankers could occur at $-400,-300,-200,-100$, $0,+100,+200$, or $+300 \mathrm{~ms}$ relative to the target. The flankers were presented for $100 \mathrm{~ms}$ and appeared only at one time point on each trial. In the no-flanker trials, only the cue and the target were shown. The trial ended when a response was recorded. Feedback was given at the end of each trial by changing the color of the fixation cross for $500 \mathrm{~ms}$. The location of the target, the orientation of the distractor, and the target-distractor SOA were balanced between conditions and randomized across trials.

Participants were instructed to respond to the orientation of a target stimulus (horizontal or vertical) as rapidly and as accurately as possible, while ignoring the flankers. Prior to the experiment, all of the participants completed practice trials. The first five blocks of 32 practice trials involved the participants responding to the target alone. The next six blocks of 32 practice trials included the flankers. The final practice block comprised 100 trials that were exactly the same as the experimental trials, so they included the cue, target, and the flankers. Initially, participants were instructed to respond as accurately as possible; then, speed was emphasized once accuracy exceeded $90 \%$. Practice was complete once participants responded with RTs $<800 \mathrm{~ms}$ and accuracy above $75 \%$. The experiment consisted of 2,400 trials, which were divided into 24 blocks of 100 trials, with 48 trials per condition. Four additional "buffer" trials were included at the start of each block and were discarded. Each participant completed one experimental session of 4 hour duration, including regular rest breaks after each block.

Analyses In all of the experiments, trials in which gaze deviated more than $2^{\circ}$ from fixation were discarded. The two dependent measures, RT and error rate, were collapsed into a single adjusted RT (adjRT) index by dividing the median RT on correct trials by the proportion of correct responses in each experimental condition. The adjRT measure, also referred to as inverse efficiency (Townsend \& Ashby, 1983), controls for speed-accuracy trade-offs and provides an integrated measure 
Fig. 2 Stimuli used in Experiments 1 and 2. a Visual angles of targets, distractors, and placeholders, and between stimuli. b Examples of target and distractor stimuli used. c Example of neutral and arrow cues with visual angles

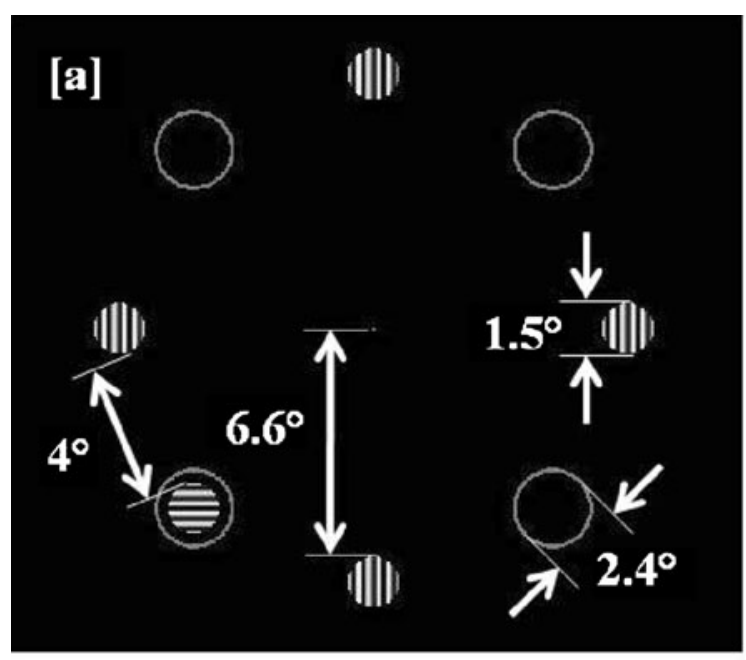

[b]

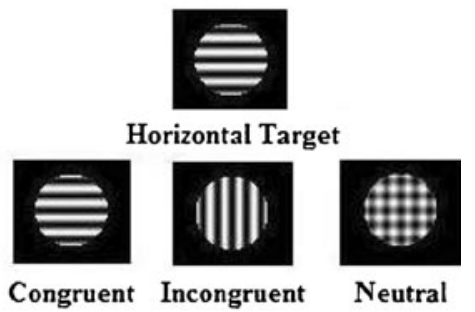

[c]

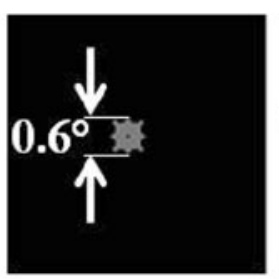

Neutral cue

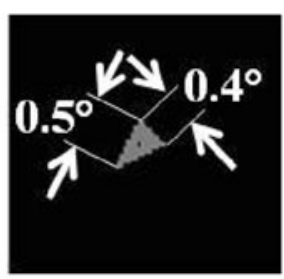

Arrow cue of performance for each participant (Chambers, Stokes \& Mattingley, 2004). Although this combination of RTs and error rates was preferred over either of the behavioral measures alone, there were in fact no speed-accuracy trade-offs in the data (see Supplementary Figs. 6-9 for separate plots of RTs and percent-correct performance). A further performance measure was the flanker effect (FE), which was calculated as $\mathrm{FE}_{\mathrm{COST}}=\operatorname{adjRT}$ on incongruent trials - adjRT on neutral trials, and $\mathrm{FE}_{\mathrm{BENEFIT}}=\operatorname{adjRT}$ on congruent trials - adjRT on neutral trials. The adjRT values for the congruent and incongruent conditions comprise performance benefits and costs due to perceptual (in)congruency (stimulus-stimulus effects) and to response (in)congruency (stimulus-response effects) of the target and the flankers. The adjRT on neutral trials, on the contrary, is caused only by perceptual incongruency, since neutral flankers have no associated response. Thus, by subtracting the adjRT on neutral trials from that on incongruent trials, the stimulus-stimulus effect is eliminated, isolating the stimulus-response effect. This subtraction thus allows us to speak of the flanker effect in terms of response competition rather than a mixture of response competition and perceptual (in)compatibility (Eriksen \& Eriksen, 1974; Machado, Wyatt, Devine \& Knight, 2007).

Data were analyzed by means of a $3 \times 2 \times 8$ repeated measures ANOVA ${ }^{1}$ with factors of flanker congruency (con-

\footnotetext{
${ }^{1}$ A Huynh-Feldt correction for violation of the sphericity assumption of ANOVA was applied where indicated by a $*$ next to the $d f$.
}

gruent, incongruent, neutral), cuing (cued, uncued) and targetflanker SOA $(-400,-300,-200,-100,0,+100,+200,+300 \mathrm{~ms})$. Additionally, we performed a paired-samples $t$ test to confirm the behavioral effect of cuing independently of the other experimental factors.

\section{Results}

All interactions and main effects of the three-way repeated measures ANOVA were found to be significant (Table 1 and Fig. 3a), thus warranting further exploration of the data via specific comparisons.

Table 1 Statistical results for the omnibus three-way repeatedmeasures ANOVA for Experiment 1. Factors are Cueing (cued, uncued), Flanker Congruency (congruent, incongruent, neutral) and Target-Flanker SOA $(-400,-300,-200,-100,0,+100,+200,+300 \mathrm{~ms})$

\begin{tabular}{llll}
\hline Effect & $F$ & $d f$ & $p$ \\
\hline Cuing & 105.68 & 1,17 & $<.001$ \\
Congruency & 18.15 & $1.2,20.2^{*}$ & $<.001$ \\
SOA & 15.94 & $4.0,68.5^{*}$ & $<.001$ \\
Cuing x Congruency & 6.89 & 2,34 & $<.01$ \\
Cuing x SOA & 5.17 & 7,119 & $<.001$ \\
Congruency x SOA & 4.25 & $4.9,82.6^{*}$ & $<.01$ \\
Cuing x Congruency x SOA & 2.45 & $10.8,183.6^{*}$ & $<.01$ \\
\hline
\end{tabular}


Cuing effect A paired-samples $t$ test comparing cued and uncued "no flanker" trials confirmed that cuing was effective in reducing adjRT, thus providing first confirmation that the main experimental manipulation was successful (adjRT cued, $M=566 \mathrm{~ms}, S D=74 \mathrm{~ms}$; adjRT uncued, $M=598 \mathrm{~ms}, S D=86 \mathrm{~ms}), t(17)=2.76, p<.05$.

To assess the effects of cuing at different target-flanker SOAs, a two-way repeated measures ANOVA with factors cuing and SOA was performed on trials with neutral flankers only (Fig. 3b). As anticipated, cuing significantly reduced adjRTs at all target-flanker SOAs [main effect cueing, $F(1,17)=71.02, p<.001]$. The (perceptual) interference of the flankers with task performance gradually changed with target-flanker SOA (Fig. 3b). Notably, at the point of target-flanker simultaneity ( $\mathrm{SOA}=0 \mathrm{~ms}$ ), the interference of the flankers on cued and uncued trials with task performance was most pronounced [main effect SOA: $\left.F\left(5.0,85.7^{*}\right)=14.98, p<.001\right]$. The pattern of performance over the eight SOAs was comparable between cued and uncued conditions; thus, the interaction of cuing and SOA did not reach statistical significance.

Flanker costs $\mathrm{FE}_{\mathrm{COST}}$ (incongruent - neutral) was submitted to a two-way repeated measures ANOVA with the factors cuing and SOA (Fig. 3c). The main effects of cuing and SOA were significant, $F(1,17)=9.43, p<.01$, and $F\left(4.2,71.1^{*}\right)=6.15, p<.001$, respectively. Post hoc single-sample $t$ tests revealed that incongruent flankers incurred a significant $\mathrm{FE}_{\mathrm{COST}}$ at target-flanker SOAs of -400 to $+100 \mathrm{~ms}$ ( $p<.05$ for all tests, collapsed across cuing). A significant cuing $x$ SOA interaction, $F(5.8,98.2 *)=2.73$, $p<.05$, indicated that the effect of attentional cuing on the $\mathrm{FE}_{\mathrm{COST}}$ was modulated by the target-flanker SOA. Post hoc paired-samples $t$ tests revealed that the $\mathrm{FE}_{\mathrm{COST}}$ was significantly reduced by cuing at target-flanker SOAs of $0 \mathrm{~ms}$ by $83 \mathrm{~ms}(p<.01)$ and $+200 \mathrm{~ms}$ by $22 \mathrm{~ms}(p<.05)$ and marginally reduced at SOA $+100 \mathrm{~ms}$ by $38 \mathrm{~ms}(p=.06)$.

Flanker benefits The $\mathrm{FE}_{\mathrm{BENEFIT}}$ (neutral - congruent) was also submitted to a two-way repeated measures ANOVA with the factors cuing and SOA (Fig. 3c). Unlike the $\mathrm{FE}_{\mathrm{COST}}$, the ANOVA of the $\mathrm{FE}_{\mathrm{BENEFIT}}$ did not reveal any significant main effects or interactions between cuing and SOA (all $F_{\mathrm{S}}<1.3$, all $p \mathrm{~s}>.25$ ).

\section{Summary}

The results of Experiment 1 are consistent with the findings of Eriksen \& Schultz (1979). For neutral flanker trials, cuing had the anticipated effect of facilitating performance at all SOAs (Posner, 1980; Posner \& Cohen, 1984).
The most pronounced effects of flankers on adjusted RTs were found for incompatible flankers. Adjusted RTs on uncued trials were slowed by incompatible flankers at SOAs from -400 to $+100 \mathrm{~ms}$. The continuous flow model (Eriksen \& Schultz, 1979) predicts precisely this behavior, because flankers preceding, co-occurring with, and shortly following the target have the capacity to prime a response incompatible with that required by the target and hence incur a behavioral cost.

Importantly, the results of Experiment 1 suggest that cuing can reduce the flanker effects. Previous work has shown that providing spatial certainty about the target location through a spatial cue can diminish the incompatible flanker effect. However, this has been shown only for exogenous cues and the simultaneous presentation of targets and flankers (Paquet \& Lortie, 1990). Our results show that an endogenous spatial cue also reduces the flanker cost, but only if the target and flankers are presented simultaneously, or if the flankers follow the target in relatively close succession.

As in the study of Eriksen and Schultz (1979), no significant benefit of compatible flankers on adjusted RT was observed at any target-flanker SOA. Unsurprisingly, we found that cuing the target location did not alter this lack of a flanker benefit. A possible reason for this lack of flanker benefits is the large target-flanker separation; we address this in more detail in the General Discussion section.

In Experiment 1, we established that endogenous selective attention can help to resolve interference from competing flankers, with the maximal attentional benefit (79\%) arising when targets and flankers occur simultaneously. These findings raise the question of where these effects arise in the information processing hierarchy. In Experiment 2, we therefore asked whether cued selective attention has an impact on measures of response competition, such as the flanker effect, if attention is initiated only prior to target onset or, alternatively, whether cuing can modulate response conflict when processing has already started.

\section{Experiment 2}

In Experiment 1, the effect of a single precue SOA on the flanker effect was examined. In Experiment 2, we assessed whether only an endogenous cue preceding the target (precue) and flankers would affect the flanker effect, or whether the same cue appearing soon after the target and flanker (postcue) could also be effective in resolving response competition. This comparison of cue onset times was intended to assess whether directing attention to the target could still reduce response competition when processing is already advanced, and response-selection processes have already started. 
(a)

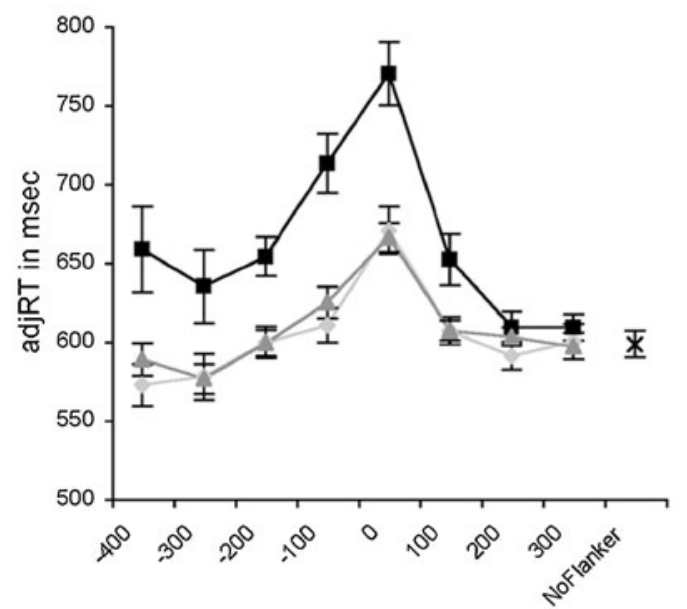

Target-Flanker SOA in msec

\section{Cued}

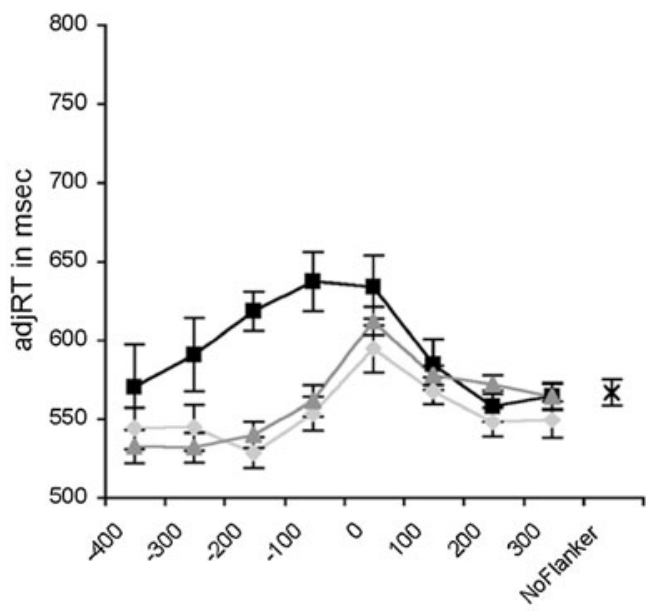

Targe-Flanker SOA in msec

(b)

\section{Cueing Effect on Neutral Trials}

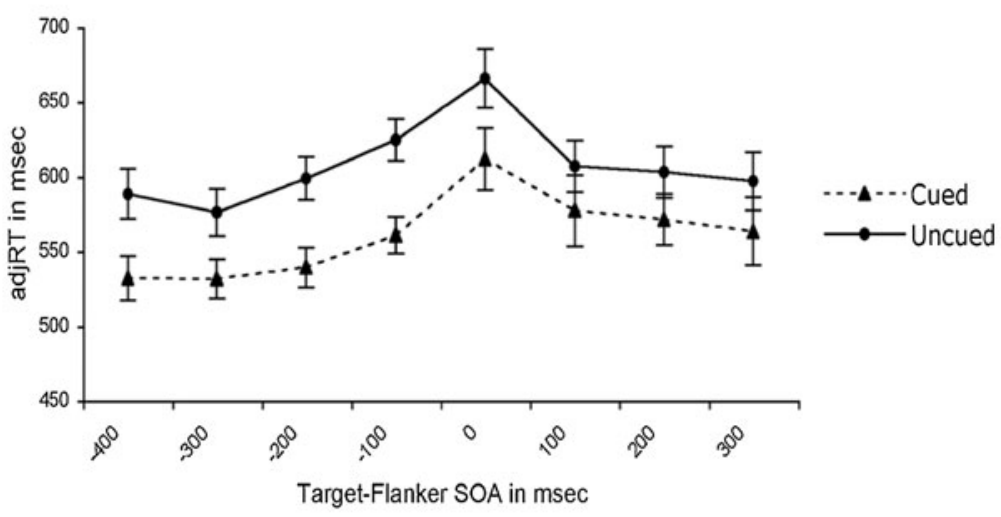

(c)

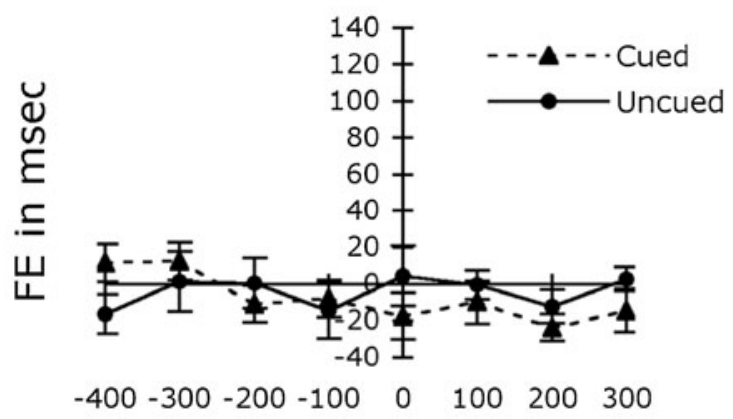

Target-flanker SOA in msec

Fig. 3 Results of Experiment 1. a Adjusted RTs in all experimental conditions. The left panel shows the results for uncued trials, the right panel shows the results for cued trials. b Adjusted RTs plotted separately for neutral trials at different target-flanker SOAs. The neutral trials isolate perceptual from response selection effects on performance. Cuing significantly reduced the adjRT at all target-flanker SOAs. c Flanker
FE Cost

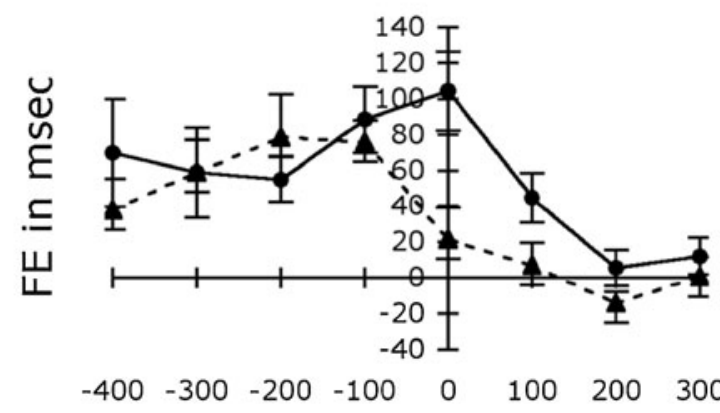

Target-flanker SOA in msec

benefits and costs at different target-flanker SOAs. Flanker benefits and costs were calculated as the difference in adjusted RTs between congruent and neutral trials, and between incongruent and neutral trials, respectively. Cuing had no significant effect on $\mathrm{FE}_{\text {BENEFIT. Cuing significantly }}$ reduced the $\mathrm{FE}_{\mathrm{COST}}$ at target-flanker SOAs between $0 \mathrm{~ms}$ and $+200 \mathrm{~ms}$. Error bars in all graphs are \pm 1 standard errors of the means 
The effects of exogenous pre- and postcues have been examined by Huckauf and Heller (2002, Experiment 2) and were discussed in the introduction. In summary, the authors found evidence that postcuing can be effective.

We thus hypothesized that an endogenous postcue would also be effective in reducing the flanker effect, provided the cue is presented sufficiently soon after the target. If the cue is presented too late after the target, stimulus processing will have proceeded to a point at which novel information - that is, the cue - can no longer interact with the selection or execution of the response. We used a procedure similar to that in Experiment 1 to replicate the effect of the $1000 \mathrm{~ms}$ precue and to investigate the effectiveness of four different postcue SOAs on the flanker effect when targets and flankers were presented simultaneously.

\section{Method}

Participants Eighteen participants, four of whom had previously taken part in Experiment 1, took part in Experiment 2 for payment. The age range was 20-26 years ( $M=21.6, S D=1.7,15$ females, three males). All participants were right-handed and had normal or correctedto-normal vision. Each participant gave his or her informed consent to participate in this study and was naive to the purpose of the experiment.

Stimuli, apparatus. and procedure The stimuli and apparatus used in the second experiment were the same as those used in Experiment 1.

The task procedure is illustrated in Fig. 4. The experimental trials began with a fixation stimulus for
$200 \mathrm{~ms}$, followed by a fixed period of $1,000 \mathrm{~ms}$ before the flankers and the target were shown simultaneously for $100 \mathrm{~ms}$. The cue was shown for $100 \mathrm{~ms}$ and could occur either $1,000 \mathrm{~ms}$ prior to the target and flankers or 100,150, 200 , or $250 \mathrm{~ms}$ afterward. At times when no other stimulus was present, the fixation point and placeholders were shown. As in Experiment 1, each trial ended when a response was recorded. To ensure sufficient time to switch from a postcue condition to a $-1,000 \mathrm{~ms}$ precue condition on the next trial, an intertrial interval of $400 \mathrm{~ms}$ was included. In Experiment 2, the participants responded in the same manner as in the first experiment, using the keypad buttons 1 and 2. Stimulus-response mapping was counterbalanced.

All of the participants completed practice trials, until they responded both quickly and accurately, as in Experiment 1 . The practice trials consisted of two blocks of 40 trials, which included only flankers and a target, and a third block of 50 trials, which included informative cues. There were a total of 1,200 experimental trials, which were split into 12 blocks of 100 trials (again including four discarded "buffer" trials per block), with 40 observations per condition. Prior to each experimental block, the participants were reminded that the arrowhead cue could appear before or after the target, and that they should attend to the cue in all conditions. Each participant undertook one session of 2 hours, with a rest break after each block of trials.

Analyses We used a $3 \times 2 \times 5$ repeated measures ANOVA with factors of flanker congruency (congruent, incongru-
Fig. 4 Stimulus sequence for the flanker task employed in Experiment 2. For details, see main text

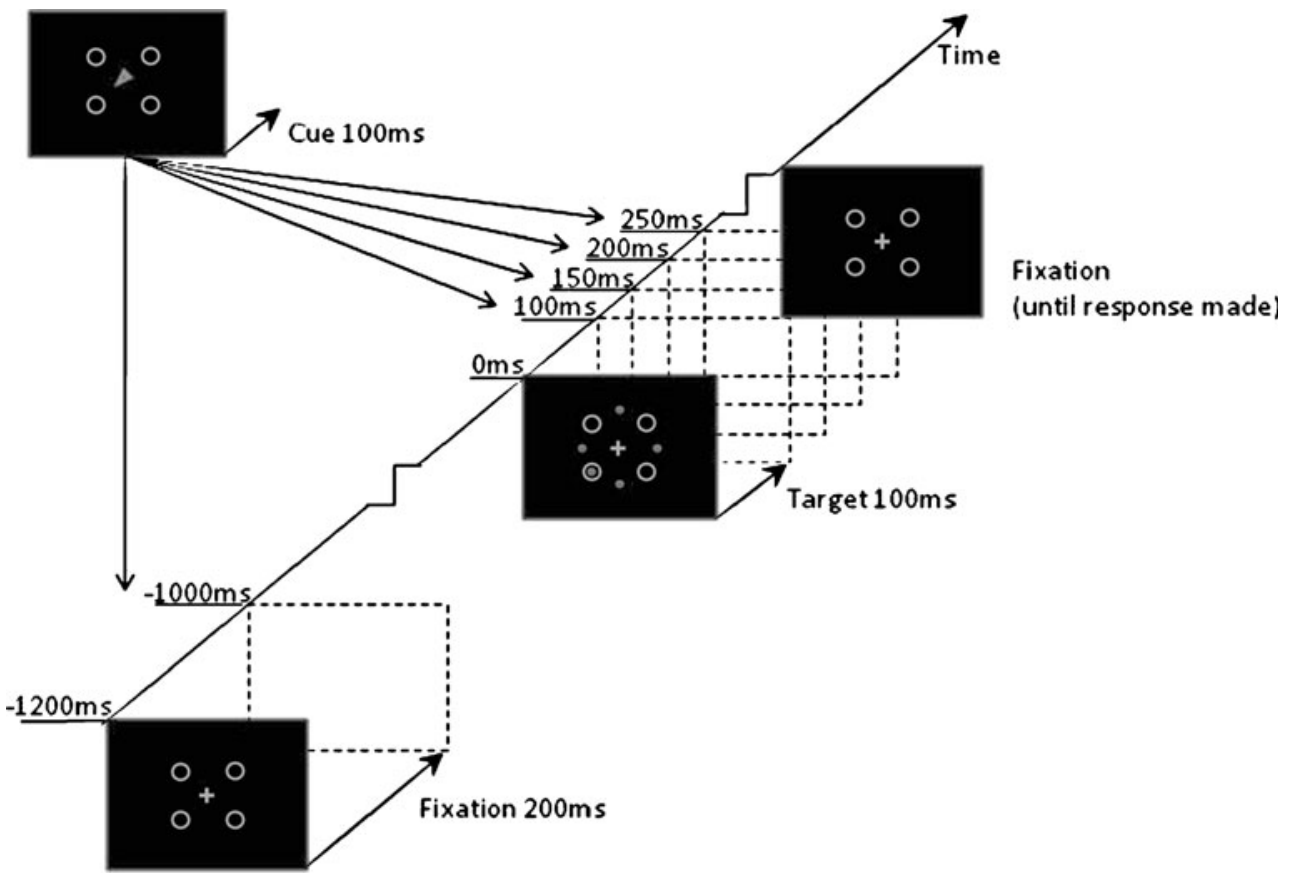


ent, neutral), cuing (cued, uncued), and cue-target SOA $(-1000,+100,+150,+200,+250 \mathrm{~ms})$. All factors were randomized from trial-to-trial within blocks, and response mapping was counterbalanced between participants. As in Experiment 1, the performance measures were adjRT and FE.

\section{Results}

All results are reported in Table 2 and Fig. 5. Importantly, the main effect of congruency, as well as the interactions of cueing $\mathrm{x}$ SOA and cuing $\mathrm{x}$ congruency $\mathrm{x}$ SOA were significant, justifying more detailed statistical comparisons.

Cuing effect In order to further explore the effect that cuing had at different cue-target SOAs, we investigated this effect on trials with neutral flankers only, thus isolating the effect of attention from stimulus-response interference (Fig. 5b). A two-way repeated measures ANOVA was conducted on neutral flanker trials only with the factors cuing and cuetarget SOA. The interaction of cuing and SOA was significant, $F(4,68)=4.17, p<.01$. Post hoc pairedsamples $t$ tests showed that cuing at an SOA of $-1,000 \mathrm{~ms}$ facilitated performance (38-ms benefit; $p<.05)$, whereas cuing at an SOA of $+100 \mathrm{~ms}$ impaired performance (48-ms cost; $p<.05)$. Cuing was ineffective at all other SOAs.

Flanker costs The effect of $\mathrm{FE}_{\mathrm{COST}}$ at different cue-target SOAs was initially assessed via a two-way repeated measures ANOVA with factors of cuing and cue-target SOA (Fig. 5c). The main effect of cuing was found to be marginally significant in benefiting behavior, $F(1,17)=$ $4.11, p=.059$, and the interaction of cuing and SOA was also significant, $F(4,68)=3.51, p<.05$. This interaction was driven by the significant reduction in the $\mathrm{FE}_{\mathrm{COST}}$ at the cue-target SOA of $-1,000 \mathrm{~ms}(p<.001$; Fig. 5 c), but not at any of the post-target SOAs (all $p \mathrm{~s}>.21$ ).

Table 2 Statistical results for the omnibus three-way repeated measures ANOVA for Experiment 2. Factors are Cueing (cued, uncued), Flanker Congruency (congruent, incongruent, neutral) and Cue-Target SOA $(-1000,+100,+150,+200,+250 \mathrm{~ms})$

\begin{tabular}{llll}
\hline Effect & $F$ & $d f$ & $p$ \\
\hline Cuing & 1.10 & 1,17 & .31 \\
Congruency & 28.476 & $1.3,21.3^{*}$ & $<.001$ \\
SOA & 18.59 & 4,68 & $<.001$ \\
Cuing x Congruency & 3.19 & $1.9,28.1^{*}$ & .065 \\
Cuing x SOA & 16.49 & 4,68 & $<.001$ \\
Congruency x SOA & 0.33 & 8,136 & .95 \\
Cuing x Congruency x SOA & 2.91 & 8,136 & $<.01$ \\
\hline
\end{tabular}

To assess whether the main effect of cuing and the interaction of cuing with SOA were, indeed, driven by the $-1,000$-ms SOA, another repeated measures ANOVA was undertaken, excluding the $-1,000 \mathrm{~ms}$ SOA. None of the main effects nor the interaction was significant, thereby confirming that only precuing affected the $\mathrm{FE}_{\mathrm{COST}}$ (all $F \mathrm{~s}<0.95$, all $p \mathrm{~s}>.44$ ).

Flanker benefits In a final analysis, the $\mathrm{FE}_{\text {BENEFIT }}$ was explored by means of a two-way repeated measures ANOVA with factors of cuing and cue-target SOA (Fig. 5c). Whereas cuing was found to be beneficial overall, as assessed by the main effect of cuing, $F(1,17)=10.51$, $p<.01$, it was not found to have any specific effects at different cue-target SOAs (nonsignificant interaction of cue $\mathrm{x}$ cue-target SOA). Post hoc paired-samples $t$ tests revealed that although there was a cuing benefit when all cue-target SOAs are pooled, such benefits were weak and were not observed reliably at any single SOA (all $p \mathrm{~s}>.087$ ).

\section{Summary}

In Experiment 2, we asked whether endogenous allocation of attention to the target location could facilitate performance on the flanker task when the shift of attention is initiated after the target appears. Late cuing effects would suggest that attentional modulation can aid in the resolution of competition even when initial processing of flankers has already occurred.

An analysis of trials with only neutral flankers showed that a precue at $-1,000 \mathrm{~ms}$ facilitated task performance as expected, whereas a postcue at $+100 \mathrm{~ms}$ worsened task performance; postcues at all other SOAs were ineffective in altering task performance when the flankers were neutral. The same improvement and worsening of task performance was found when congruent or incongruent flankers were employed. However, a postcue at $+100 \mathrm{~ms}$ had the same effect on trials with congruent or incongruent flankers as on trials with neutral flankers. Thus, no significant cuing benefits or costs were observed at $+100 \mathrm{~ms}$. In fact, flanker benefits did not increase reliably at any single cue-target SOA, although a main effect of cuing was observed. Possible reasons for the lack of a cuing effect on flanker benefits are covered in the General Discussion section. Flanker costs were decreased only by precuing and were unaffected by cuing at all other SOAs.

Our experimental design did not provide a definitive explanation for the generalized performance cost at $+100 \mathrm{~ms}$, but a likely reason is attentional capture by the central cue (Ansorge, Horstmann \& Carbone, 2005; Belopolsky, Schreij \& Theeuwes, 2010; Burnham, 2007; Eimer \& Kiss, 2008; Eimer, Kiss, Press \& Sauter, 2009; Folk, Remington, \& Johnston, 1992; Gibson \& Kelsey, 1998). We found that informative, 
(a)
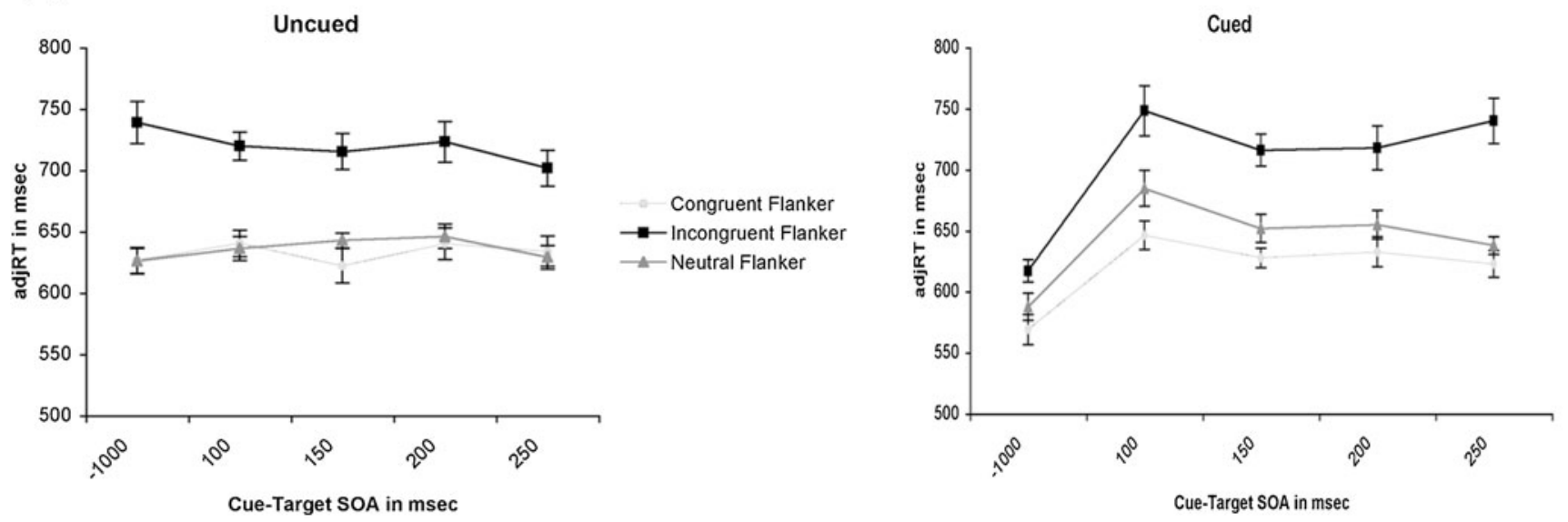

(b)

Cueing Effect on Neutral Trials

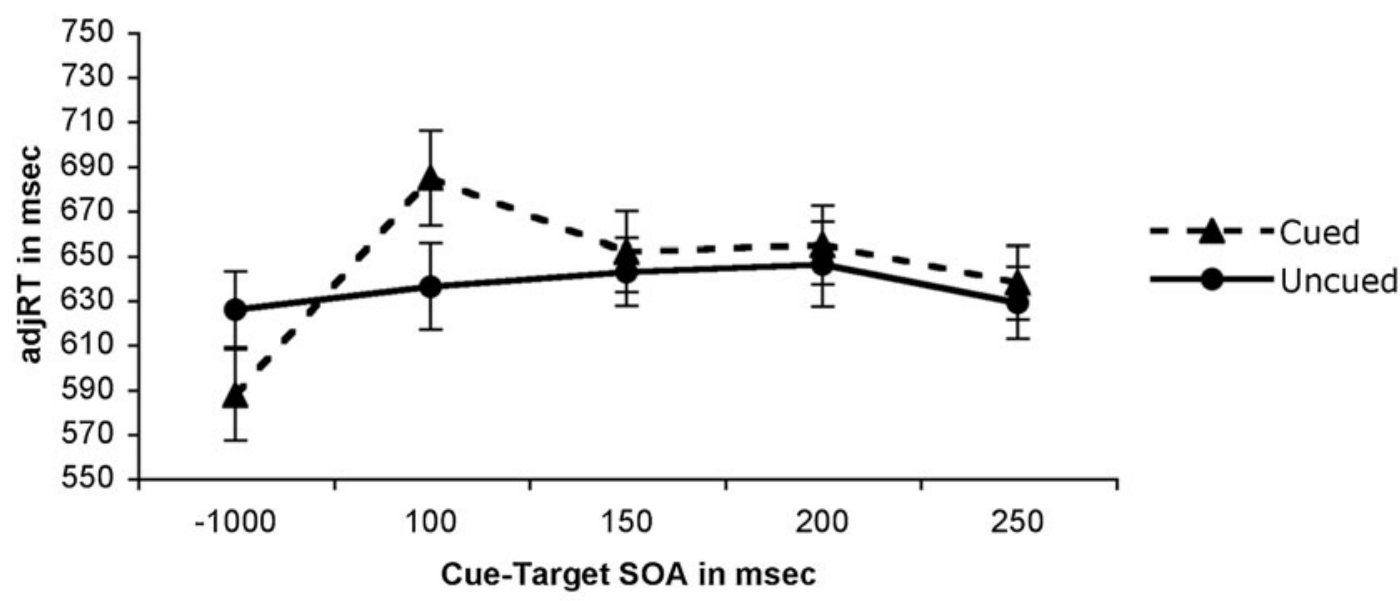

(c)

FE Benefit

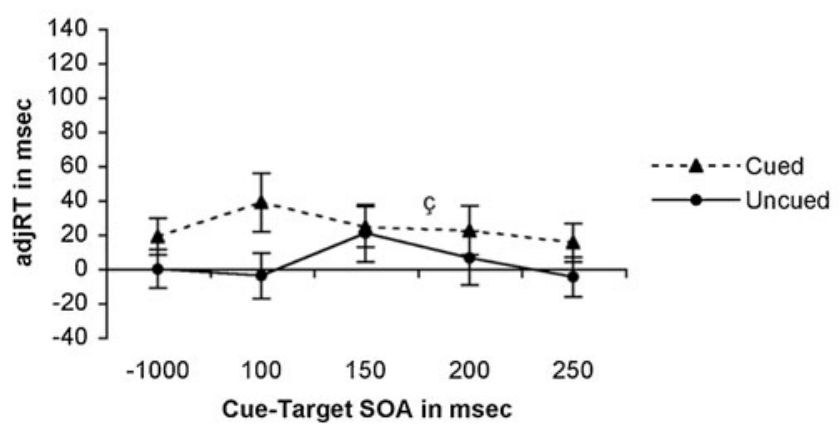

Fig. 5 Results of Experiment 2. a Adjusted RTs in all experimental conditions. The left panel shows the results for uncued trials, the right panel shows the results for cued trials. b Adjusted RTs plotted separately for neutral trials at each of the target-flanker SOAs. The neutral trials isolate perceptual from response selection effects on performance. Cuing benefited performance at a cue-target SOA of $-1,000 \mathrm{~ms}$, but impaired performance at a cue-target SOA of $+100 \mathrm{~ms}$. c Flanker benefits and costs at different target-flanker SOAs. Flanker benefits and
FE Cost

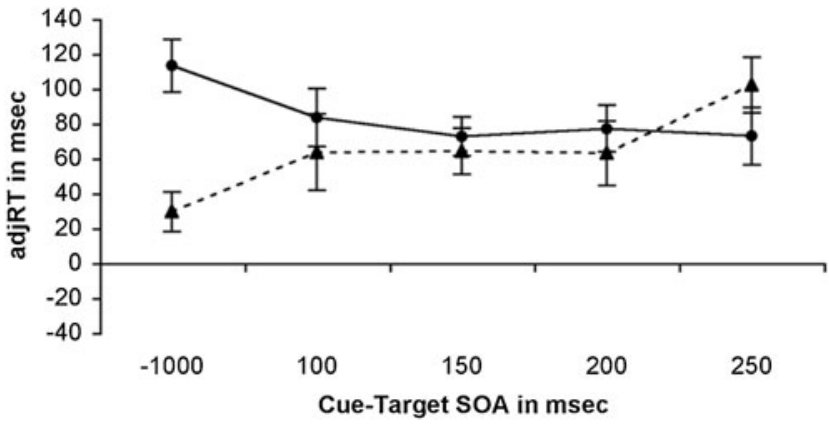

costs were calculated as the difference in adjusted RTs between congruent and neutral trials, and between incongruent and neutral trials, respectively, and reflect effects of response selection on performance. Cuing increased the $\mathrm{FE}_{\mathrm{BENEFIT}}$ overall, but not significantly at any single cue-target SOA. Cuing significantly reduced the $\mathrm{FE}_{\mathrm{COST}}$ only at a cue-target SOA of $-1,000 \mathrm{~ms}$. Error bars in all graphs are \pm 1 standard errors of the means 
but not uninformative, postcues led to a performance cost at $+100 \mathrm{~ms}$. It is possible that participants redirected their attention to the cue or split their attention between the cue and the target location, thus entering into a situation of divided attention. Attention was not redirected to or shared with noninformative cues, thus resulting in no divided attention cost. Attention might have been captured only by task-relevant, informative cues according to the principles of contingent attentional capture. The performance cost incurred by all informative cues is thus likely a general cost due to divided attention and can be distinguished from an effect of cuing on response selection.

The fact that a generalized cuing cost is not incurred at longer postcuing SOAs indicates that the cost is, indeed, incurred at a perceptual stage of processing, rather than at a response selection stage.

Thus, the results of Experiment 2 are in accordance with the hypothesis that only endogenous pre- but not postcues are effective in altering task performance in response competition tasks. This suggests that the benefit of endogenous attention in reducing the behavioral cost of incongruent flankers originates from boosting the perceptual representation of the target during early initial processing stages.

\section{General discussion}

Selective attention and response selection are two crucial functions in overt behavior. Although the temporal processing of each of these functions has been studied individually (Cheal \& Lyon, 1989; Cheal \& Lyon, 1991; Eriksen \& Schultz, 1979; Flowers, 1980; Flowers, 1990; Flowers \& Wilcox, 1982; Grice et al., 1984; Müller \& Rabbitt, 1989; Stoffer, 1993; Taylor, 1977; Wascher et al., 1999), to date little is known about the temporal interaction of these related functions (Paquet \& Lortie, 1990; Ro et al., 2002). In two experiments, we studied how selective attention can aid response selection. To this end, we combined a selective attention task with an Eriksen flanker response selection task. The effect of attentional cuing on the flanker effect was studied for different target-flanker SOAs in Experiment 1 . It was found that attention modulated response competition when targets and flankers were presented simultaneously or when the flanker closely followed the target. The results of Experiment 2 showed that directing attention endogenously to the target location before, but not after, target presentation improved performance on the flanker task. These results reveal that endogenous attention influences response competition only when attention is cued during early perceptual processing levels; once target and flankers had been identified, endogenous cuing seemed to have little effect.
Attentional cuing of target locations has been studied extensively, and it is well established that an endogneous cue such as ours, appearing 1,000 ms before the target, affects performance on simple perceptual detection tasks (Cheal \& Lyon, 1989; Cheal \& Lyon, 1991; Müller \& Rabbitt, 1989; Posner, 1980; Posner \& Cohen, 1984; Stoffer, 1993). Whether or not attentional cuing can affect performance on a response competition task, such as the flanker task, however, is debated (Paquet \& Lortie, 1990; Ro et al., 2002). Consistent with Paquet and Lortie, who used exogenous cues, the flanker costs in our study were significantly decreased by an endogenous attentional cue. Thus, it appears that both endogenous and exogenous attention can interact with measures of response competition. Ro et al. failed to find such an interaction in a series of four experiments. In light of the evidence from our and the two previous positive results, it seems likely that Ro et al.'s null results were due to experimental factors. As discussed in the introduction, such factors could be that (1) the target location was always known, (2) only one flanker stimulus was used, (3) cuing was not fully informative, (4) only simultaneous target-flanker presentations were tested, (5) the number of trials per participant was relatively low, and (6) the flanker, rather than the target location, was cued.

In Experiment 1, we found that cuing decreased the flanker costs only if the target and flanker were presented simultaneously or in close positive succession to each other $(+100 \mathrm{~ms}$ and $+200 \mathrm{~ms})$; at all other target-flanker SOAs, the cue was ineffective. This finding is consistent with results from contingent attentional capture experiments. Attentional capture is the potential of task-irrelevant information to exogenously direct attention under circumstances of goal-directed endogenous attention. Contingent attentional capture occurs when the task-irrelevant stimulus contains task-defining features, as was the case with our flankers. It has been found that contingent attentional capture can easily override goal-directed attention (Ansorge et al., 2005; Belopolsky et al., 2010; Burnham, 2007; Eimer \& Kiss, 2008; Eimer et al., 2009; Folk et al., 1992; Gibson $\&$ Kelsey, 1998). We suggest that flankers preceding the target capture attention, and this involuntary process is unchanged by endogenous cuing. When flankers are presented simultaneously or slightly later than the target, the cue prioritizes target selection, strongly suppressing attentional capture by the flankers.

Thus, the results of Experiment 1, together with previous findings (Paquet \& Lortie, 1990), indicate that selective attention can interact with a response competition task. However, at what level of information processing this interaction takes place could not be answered from the results of Experiment 1; this was the focus of our second experiment, in which we examined whether endogenous cuing would still influence the flanker effect if the cue were 
presented after the target appeared. Our analysis of only the neutral flanker trials shows that cuing did affect the perceptual component of the flanker effect: At $-1,000 \mathrm{~ms}$, the cue produced the expected performance benefit. At $+100 \mathrm{~ms}$, however, the cue incurred a performance cost. Our experimental design does not provide a definitive explanation for the performance cost at $+100 \mathrm{~ms}$, but a likely reason is again attentional capture: A behaviorally relevant stimulus (the cue) appeared while participants were directing their attention to a peripheral target. This situation of divided attention impaired performance. This is not an impairment of response selection, however. Response selection impairments would be reflected in increased flanker costs at $+100 \mathrm{~ms}$, but no such increase was found. Thus, the endogenous direction of selective attention does not appear to have an effect on response selection at any of the postcuing SOAs.

In Experiment 2, precuing as well as postcuing appear to have had a modest general effect on increasing the flanker benefit; however, this result needs further investigation, since no single cue-target SOA by itself significantly increased the flanker benefit. The effect of attentional cuing on the flanker costs was very clear: Precuing, but not postcuing, reduced the flanker cost. This result provides clear evidence about the critical time period at which attention interacted with our measure of response competition. Thus, preparatory attention can interact with response competition. However, rather than reflecting a direct influence of attention at a response-selection stage, this benefit could have arisen because of modulation of perceptual processes during the initial forward sweep of information processing (Lamme \& Roelfsema, 2000). Cues, task-sets, or expectations can direct attention to a target location and thus facilitate perception, and hence behavior. However, it appears that this needs to happen prior to or rapidly after encountering the visual information in order for endogenous attention to interact with measures of response competition. Once information has entered the system, attention has very limited time to facilitate response selection by confirming a target location.

In Cohen et al.'s (1992) PDP model, selective attention aids the resolution of response competition at a perceptual level. Cohen and colleagues' model implements attention as units, which act upon the representation strengths of perceptual inputs. This model has been shown to robustly capture not only the basic findings of the Eriksen flanker task (Eriksen \& Eriksen, 1974), such as congruence benefits and incongruence costs, but also to capture more intricate and less intuitive findings (e.g., those reported by Gratton, Coles, Sirevaag, Eriksen \& Donchin, 1988). Our data demonstrate that (a) selective attention interacts with perceptual processing, and that (b) selective attention does not reduce response competition when targets and flankers are already visually processed. Thus, our data are in accord with Cohen et al.'s (1992) suggestion that visual attention does play an important role in solving response conflict, but only at the initial processing stages.

We have discussed all cuing benefits on the flanker effects in terms of attentional enhancement of the target. Load Theory (Lavie, 1995; Lavie, Hirst, de Fockert \& Viding, 2004) would suggest that the more attention is directed to a target, the less processing resources will be available to the processing of task-irrelevant (flanker) stimuli. Thus, it is entirely possible that the effects we observe rest not only on attentional enhancement of the target, but also on concomitant attentional suppression of the flankers.

Also, we do not describe our results in terms of resistance to crowding. We avoid doing so for three reasons: First, under uncued conditions, it has been found that the spatial extent of crowding is roughly half the target eccentricity (e.g., Bouma, 1970; Toet \& Levi, 1992). Because the eccentricity of our targets was $6^{\circ}$ visual angle, our closest edge-to-edge distance between stimuli of $4^{\circ}$ is unlikely to have produced significant crowding. Yeshurun and Rashal (2010) showed that spatial attention further reduces the target-flanker distance at which the flankers no longer interfere with the target identification. Second, Pelli, Palomares \& Majaj (2004) have reported that crowding affects feature detection, which was not necessary to solve our task. Instead, crowding appears to affect feature integration, which was not required in our task. Finally, the critical aspect of our flanker task was not the spatial proximity of the targets and flankers (which was held constant across all conditions) but the unique performance cost associated with resolving response conflict on incongruent versus congruent and neutral trials (for more information on crowding, see Whitney \& Levi, 2011).

In both of our experiments, significant flanker benefits were not obtained; hence, they could not be modulated by cuing. This result is presumably due to the large spatial separation between targets and flankers (edge-to-edge $4^{\circ}$ visual angle): It has been shown that target RT is little affected by simultaneous compatible noise when targetnoise separation is large enough to eliminate contour interactions $\left(>0.5^{\circ}\right.$; Eriksen \& Eriksen, 1974; Flom, Weymouth \& Kahneman, 1963). Additionally, it has been shown that although a cost resulting from incompatible flankers is commonly observed, behavioral benefits resulting from compatible flankers are less consistently found and are generally of much smaller magnitude (see Flowers, 1990, for a discussion of this finding). Thus, the existing literature suggests that it is unsurprising that we did not observe flanker benefits with our stimulus display parameters. Future research will need to show whether cuing affects flanker benefits in the same manner as it does flanker costs. 
Previous and current research confirm a role of selective attention in early, visual perceptual, processing (Cheal \& Lyon, 1989; Cheal \& Lyon, 1991; Müller \& Rabbitt, 1989; Stoffer, 1993). Little is known, however, about how selective attention can influence later response-selection processing stages (Paquet \& Lortie, 1990; Ro et al., 2002). Our results show that selective attention can aid the resolution of response competition insofar as it can perceptually prioritize relevant over irrelevant information. Our results, however, reveal no evidence that attention aids the resolution of response competition once irrelevant information has entered the system.

Author Note The present research was supported by BBSRC Grant BB/E020291/1 (to J.K. and C.D.C.) and a BBSRC David Phillips Fellowship (to C.D.C.).

\section{References}

Ansorge, U., Horstmann, G., \& Carbone, E. (2005). Top-down contingent capture by color: Evidence from RT distribution analyses in a manual choice reaction task. Acta Psychologica, 120, 243-266.

Belopolsky, A. V., Schreij, D., \& Theeuwes, J. (2010). What is topdown about contingent capture? Attention, Perception, \& Psychophysics, 72, 326-341.

Bouma, H. (1970). Interaction effects in parafoveal letter recognition. Nature, 226, 177-178.

Burnham, B. R. (2007). Displaywide visual features associated with a search display's appearance can mediate attentional capture. Psychonomic Bulletin \& Review, 14, 392-422.

Chambers, C. D., Bellgrove, M. A., Gould, I. C., English, T., Garavan, H., McNaught, E., et al. (2007). Dissociable mechanisms of cognitive control in prefrontal and premotor cortex. Journal of Neurophysiology, 98, 3638-3647.

Chambers, C. D., Stokes, M. G., \& Mattingley, J. B. (2004). Modalityspecific control of strategic spatial attention in parietal cortex. Neuron, 44, 925-930.

Chastain, G., Cheal, M., \& Lyon, D. R. (1996). Attention and nontarget effects in the location-cuing paradigm. Perception \& Psychophysics, 58, 300-309.

Cheal, M. L., \& Lyon, D. (1989). Attention effects on form discrimination at different eccentricities. Quarterly Journal of Experimental Psychology Section A: Human Experimental Psychology, 41, 719-746.

Cheal, M. L., \& Lyon, D. R. (1991). Central and peripheral precuing of forced-choice discrimination. Quarterly Journal of Experimental Psychology Section A: Human Experimental Psychology, 43, 859-880.

Cohen, J. D., Servan-Schreiber, D., \& McClelland, J. L. (1992). A parallel distributed processing approach to automaticity. The American Journal of Psychology, 105, 239-269.

Deutsch, J. A., \& Deutsch, D. (1963). Attention: Some theoretical considerations. Psychological Review, 70, 51-61.

Eimer, M., \& Kiss, M. (2008). Involuntary attentional capture is determined by task set: Evidence from event-related brain potentials. Journal of Cognitive Neuroscience, 20, 14231433.

Eimer, M., Kiss, M., Press, C., \& Sauter, D. (2009). The roles of feature-specific task set and bottom-up salience in attentional capture: An ERP Study. Journal of Experimental Psychology. Human Perception and Performance, 35, 1316-1328.

Eriksen, B. A., \& Eriksen, C. W. (1974). Effects of noise letters upon the identification of a target letter in a non-search task. Perception \& Psychophysics, 16, 143-149.

Eriksen, C. W., \& Schultz, D. W. (1979). Information processing in visual search: A continuous flow conception and experimental results. Perception \& Psychophysics, 25, 249-263.

Flom, M. C., Weymouth, F. W., \& Kahneman, D. (1963). Visual resolution and contour interaction. Journal of the Optical Society of America, 53, 1026-1032.

Flowers, J. H. (1980). Response priming effects in a digit naming task as a function of target-noise separation. Bulletin of the Psychonomic Society, 16, 443-446.

Flowers, J. H. (1990). Priming effects in perceptual classification. Perception \& Psychophysics, 47, 135-148.

Flowers, J. H., \& Wilcox, N. (1982). The effect of flanking context on visual classification: The joint contribution of interactions at different processing levels. Perception \& Psychophysics, 32, 581-591.

Folk, C. L., Remington, R. W., \& Johnston, J. C. (1992). Involuntary covert orienting is contingent on attentional control settings. Journal of Experimental Psychology. Human Perception and Performance, 18, 1030-1044.

Gibson, B. S., \& Kelsey, E. M. (1998). Stimulus-driven attentional capture is contingent on attentional set for displaywide visual features. Journal of Experimental Psychology. Human Perception and Performance, 24, 699-706.

Gratton, G., Coles, M. G., Sirevaag, E. J., Eriksen, C. W., \& Donchin, E. (1988). Pre- and poststimulus activation of response channels: A psychophysiological analysis. Journal of Experimental Psychology. Human Perception and Performance, 14, 331-344.

Grice, G. R., Boroughs, J. M., \& Canham, L. (1984). Temporal dynamics of associative interference and facilitation produced by visual context. Perception \& Psychophysics, 36, 499-507.

Hazeltine, E., Bunge, S. A., Scanlon, M. D., \& Gabrieli, J. D. (2003). Material-dependent and material-independent selection processes in the frontal and parietal lobes: An event-related fMRI investigation of response competition. Neuropsychologia, 41, 1208-1217.

Huckauf, A., \& Heller, D. (2002). Spatial selection in peripheral letter recognition: In search of boundary conditions. Acta Psychologica, 111, 101-123.

Jonides, J. (1980). Towards a model of the mind's eye's movement. Canadian Journal of Psychology 34, 103-112.

Klein, R. M. (2004). On the control of orienting. In M. I. Posner (Ed.), Cognitive neuroscience of attention (pp. 29-44). New York: Guilford Press.

Kornblum, S., Hasbroucq, T., \& Osman, A. (1990). Dimensional overlap: Cognitive basis for stimulus-response compatibility-A model and taxonomy. Psychology Review, 97, 253-270.

Lamme, V. A., \& Roelfsema, P. R. (2000). The distinct modes of vision offered by feedforward and recurrent processing. Trends in Neuroscience, 23, 571-579.

Lavie, N. (1995). Perceptual load as a necessary condition for selective attention. Journal of Experimental Psychology. Human Perception and Performance, 21, 451-468.

Lavie, N., Hirst, A., de Fockert, J. W., \& Viding, E. (2004). Load theory of selective attention and cognitive control. Journal of Experimental Psychology. General, 133, 339-354.

Machado, L., Wyatt, N., Devine, A., \& Knight, B. (2007). Action planning in the presence of distracting stimuli: An investigation into the time course of distractor effects. Journal of Experimental Psychology. Human Perception and Performance, 33, 10451061.

Müller, H. J., \& Rabbitt, P. M. A. (1989). Reflexive and voluntary orienting of visual attention: Time course of activation and 
resistance to interruption. Journal of Experimental Psychology. Human Perception and Performance, 15, 315-330.

Munneke, J., Van der Stigchel, S., \& Theeuwes, J. (2008). Cueing the location of a distractor: An inhibitory mechanism of spatial attention? Acta Psychologica, 129, 101-107.

Paquet, L., \& Lortie, C. (1990). Evidence for early selection: Precuing target location reduces interference from same-category distractors. Perception \& Psychophysics, 48, 382-388.

Pashler, H. (1994). Dual-task interference in simple tasks: Data and theory. Psychological Bulletin, 116, 220-244.

Pelli, D. G., Palomares, M., \& Majaj, N. J. (2004). Crowding is unlike ordinary masking: Distinguishing feature integration from detection. Journal of Vision, 4.

Posner, M. I. (1980). Orienting of attention. Quarterly Journal of Experimental Psychology, 32, 3-25.

Posner, M. I., \& Cohen, Y. (1984). Components of visual orienting. In H. Bourna \& D. G. Bouwhuis (Eds.), Attention and Performance X: Control of language processes (pp. 531-556). London: Erlbaum.

Ridderinkhof, K. R. (2002). Micro- and macro-adjustments of task set: Activation and suppression in conflict tasks. Psychological Research, 66, 312-323.

Ridderinkhof, K. R., \& van der Molen, M. W. (1995). When global information and local information collide: A brain potential analysis of the locus of interference effects. Biological Psychology, 41, 29-53.

Ro, T., Machado, L., Kanwisher, N., \& Rafal, R. D. (2002). Covert orienting to the locations of targets and distractors: Effects on response channel activation in a flanker task. The Quarterly Journal of Experimental Psychology. A, 55, 917-936.
Smith, P. L., \& Ratcliff, R. (2004). Psychology and neurobiology of simple decisions. Trends in Neuroscience, 27, 161-168.

Sternberg, S. (1969). The discovery of processing stages: Extensions of Donder's method. In W. G. Koster (Ed.), Attention and Performance II (pp. 276-315). Amsterdam: North-Holland Publishing Company.

Stoffer, T. H. (1993). The time course of attentional zooming: A comparison of voluntary and involuntary allocation of attention to the levels of compound stimuli. Psychological Research, 56, $14-25$.

Taylor, D. A. (1977). Time course of context effects. Journal of Experimental Psychology. General, 106, 404-426.

Toet, A., \& Levi, D. M. (1992). The two-dimensional shape of spatial interaction zones in the parafovea. Vision Research, 32, 1349-1357.

Townsend, J. T., \& Ashby, F. G. (1983). Stochastic modelling of 1240 elementary psychological processes. London: Cambridge University Press.

Treisman, A. M. (1969). Strategies and models of selcetive attention. Psychological Review, 76, 282-299.

Wascher, E., Reinhard, M., Wauschkuhn, B., \& Verleger, R. (1999). Spatial S-R compatibility with centrally presented stimuli. An event-related asymmetry study on dimensional overlap. Journal of Cognitive Neuroscience, 11, 214-229.

Whitney, D., \& Levi, D. M. (2011). Visual crowding: A fundamental limit on conscious perception and object recognition. Trends in Cognitive Science, 15, 160-168.

Yeshurun, Y., \& Rashal, E. (2010). Precueing attention to the target location diminishes crowding and reduces the critical distance. Journal of Vision, 10, 16. 